\title{
The Effect of Exercise Training on Serum Level of $\beta$-Estradiol, Testosterone, and Cognitive Deficit in Rats with Letrozole-Induced Polycystic Ovary Syndrome
}

\author{
Samaneh Rafiei ${ }^{1}$, Mohammad Amin Edalatmanesh ${ }^{2 *}$ \\ ${ }^{1}$ Department of Exercise Physiology, Marvdasht Branch, Islamic Azad University, Marvdasht, Iran \\ ${ }^{2}$ Department of Physiology, College of Sciences, Shiraz Branch, Islamic Azad University, Shiraz, Iran
}

\section{ABSTRACT}

Introduction: Polycystic ovary syndrome (PCOS) is a complex endocrine and metabolic disorder with unclear etiology, which characterized by ovulatory dysfunction and hyperandrogenism. We investigated the possible influence of exercise training on the serum level of $\beta$-estradiol and testosterone as well as on cognitive functions in rat model of PCOS. Materials and Methods: To induce PCOS, 30 virgin female Sprague dawley rats received letrozole continuously $(1 \mathrm{mg} / \mathrm{kg} / \mathrm{d})$. After 28 day of letrozole intra- peritoneal injection, rats were randomly divided into the PCOS and PCOS+exercise groups and compared with healthy controls. In PCOS+exercise group, the exercise program was to 20 minutes daily swimming for four weeks. After four weeks, we evaluated spatial memory by Morris water maze test in all groups. Then, the serum level of free testosterone and $\beta$-estradiol was measured using ELISA. Results: The findings indicated an increase of serum level of testosterone and $\beta$-estradiol with spatial memory deficits in PCOS rats. However, exercise training significantly reduced the serum level of both hormones and improved cognitive function in PCOS+exercise rats. Conclusion: These results demonstrated that shortterm exercise training can ameliorate the cognitive deficit and decrease the serum level of testosterone and $\beta$-estradiol in PCOS model. The exercise training can be the basic therapeutic means for cognitive aspect of PCOS.

\section{Key words:}

1. Gonadal Steroid Hormones 2. Polycystic Ovary Syndrome 3. Cognition

* Corresponding Author: Mohammad Amin Edalatmanesh

E-mail: amin.edalatmanesh@gmail.com 


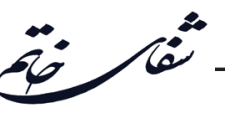

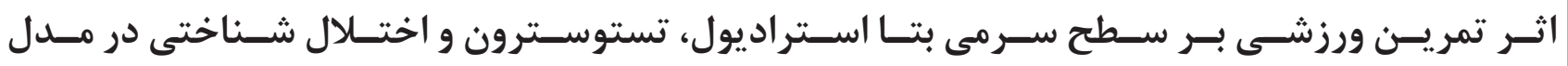

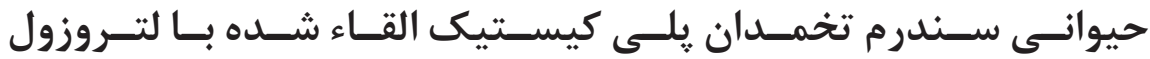

سمانه رفيعى'، محمد امين عدالت منش r."

'اكروه فيزيولوزى ورزشى، واحد مرودشت، دانشعاه آزاد اسلامى، مرودشت، ايران

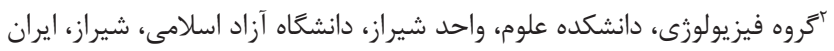

كليد وازهها:

1. ا. هورمونهاى استروئيدى كَناد

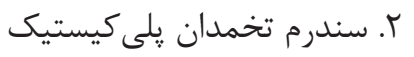

r.

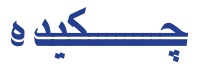

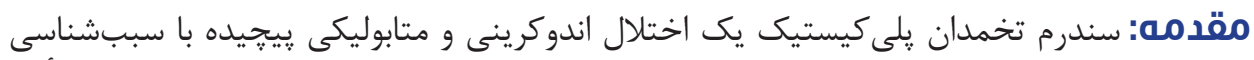

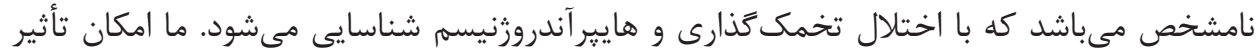

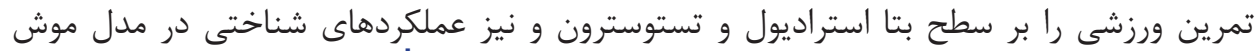

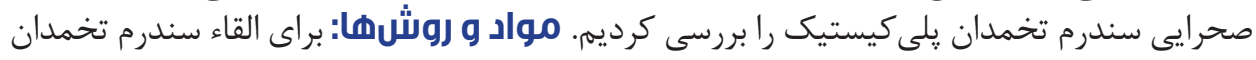

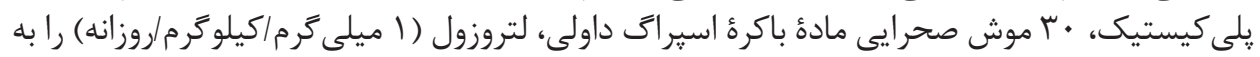

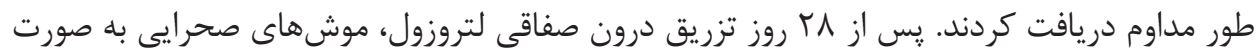

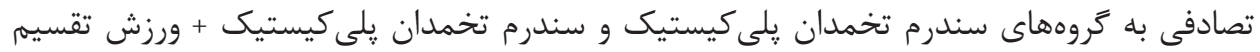

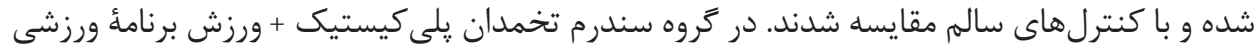

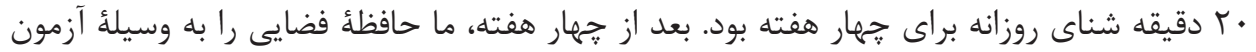

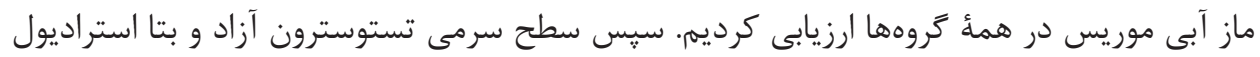

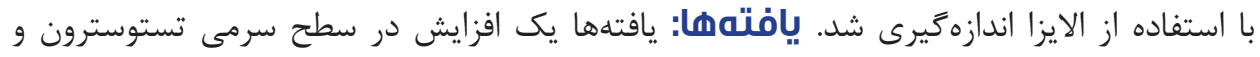

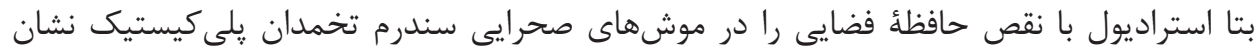

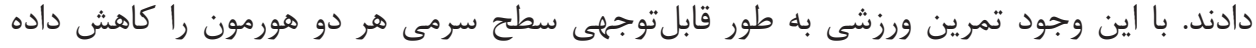

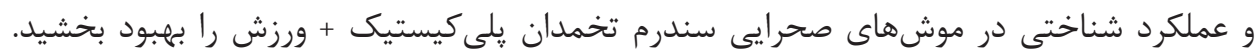

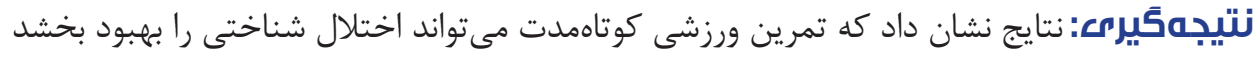

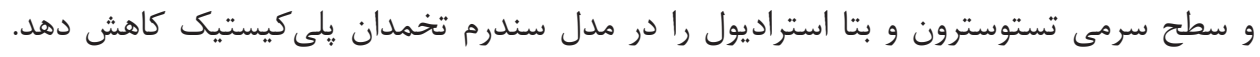

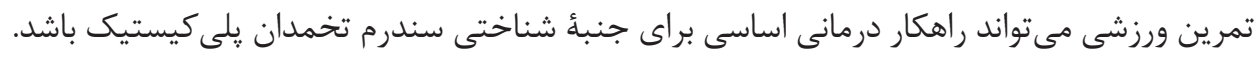

" نويسنده مسئول: محمد امين عدالت منش آدرس الكترونيكى: amin.edalatmanesh@gmail.com 


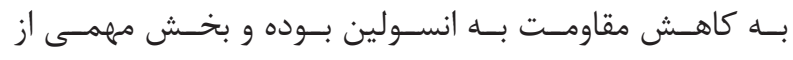

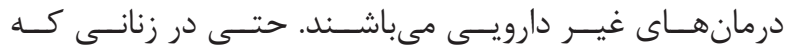

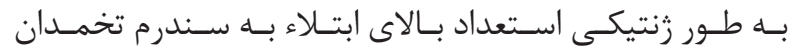

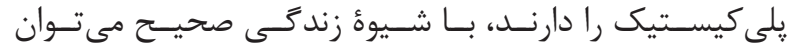

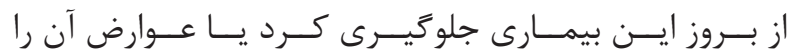

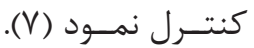

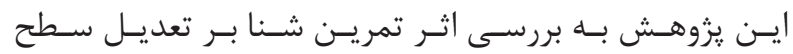

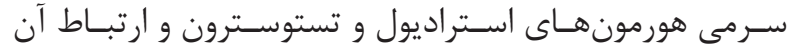

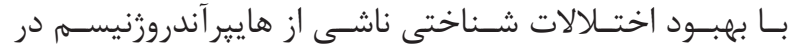

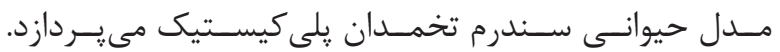

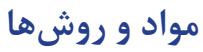
حيوانات

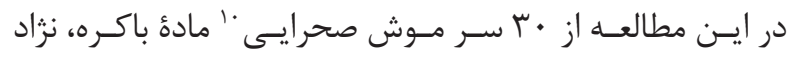

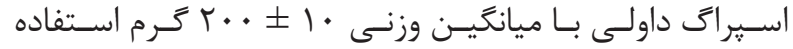

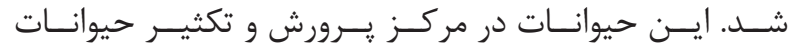

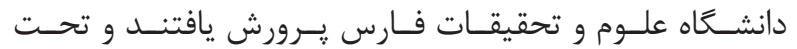

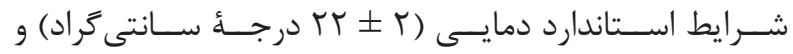

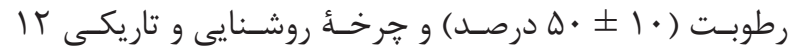

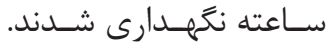

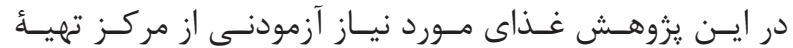

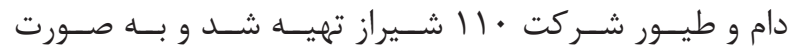

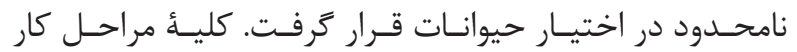

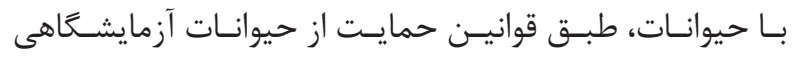

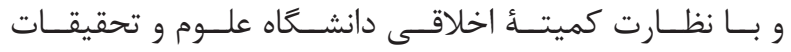

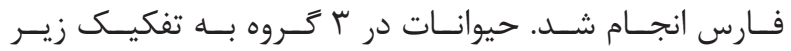

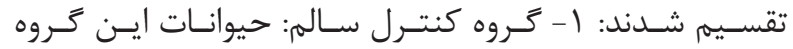

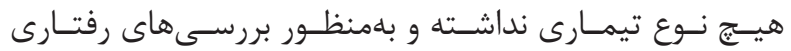

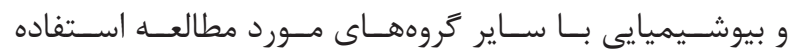

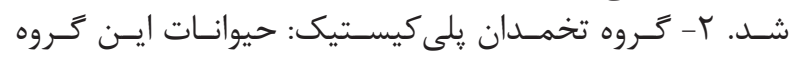

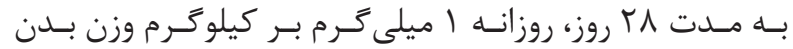

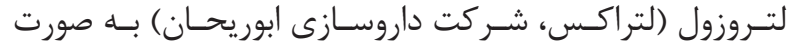

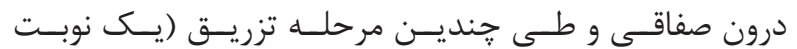

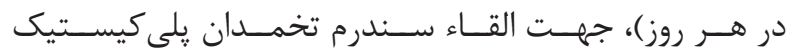

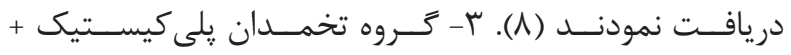

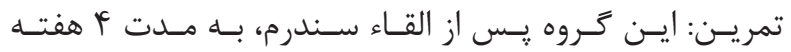
تمريسن شــنـا داشـتـند

$$
\text { آزمون اسمير وازينال }
$$

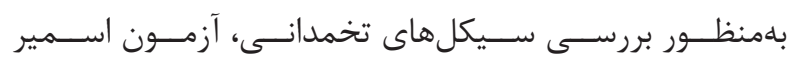

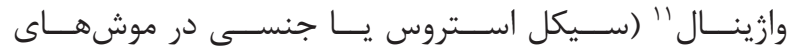

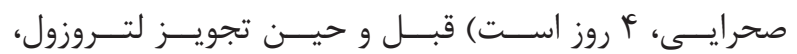

${ }^{1}$ Polycystic ovary syndrome

${ }^{2}$ Endocrine

${ }^{3}$ Premenopausal

${ }^{4}$ Hirsutism

${ }^{5}$ Androgenes

${ }^{6}$ Follicle-stimulating hormone

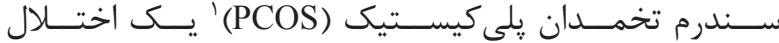

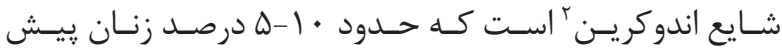

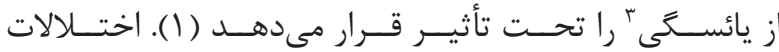

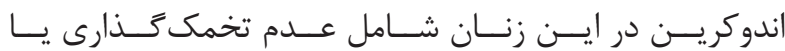

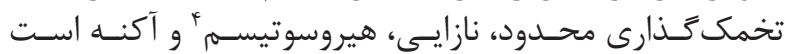

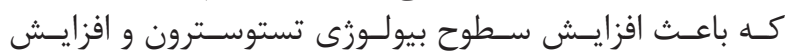

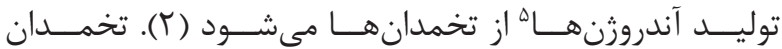

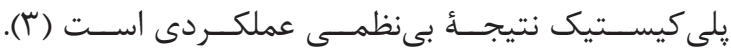

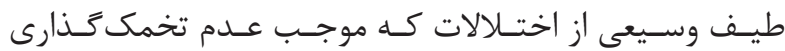

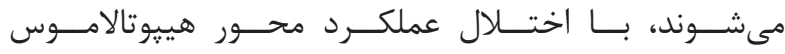

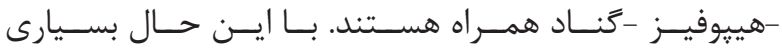

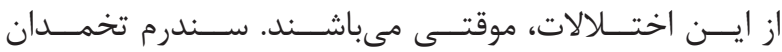

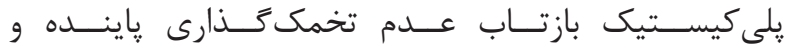

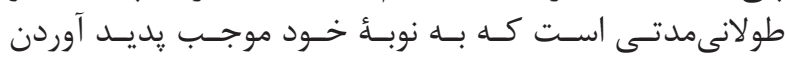

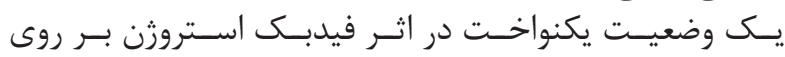

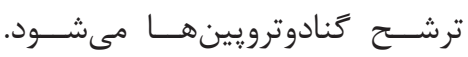

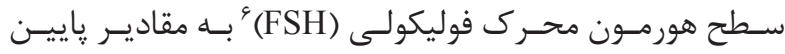

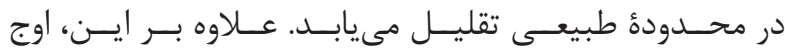

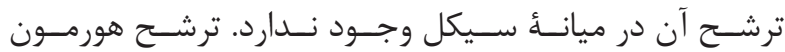

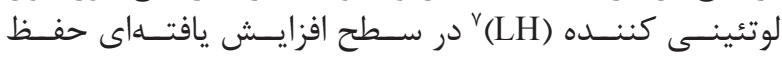

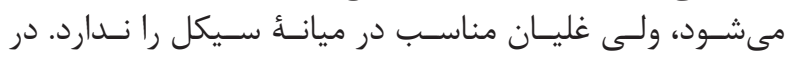

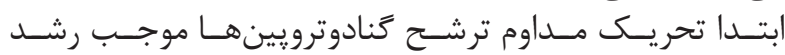

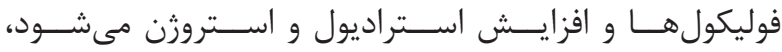

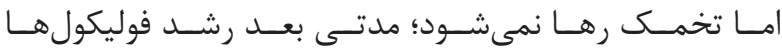

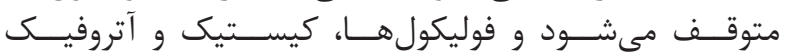

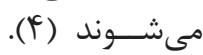

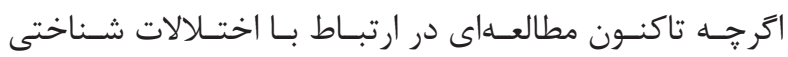

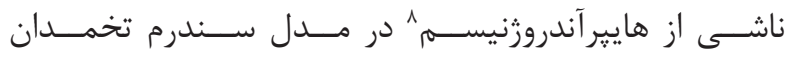

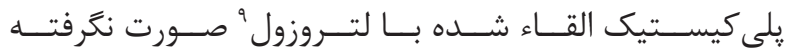

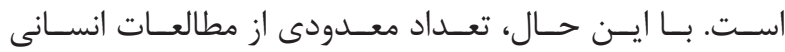

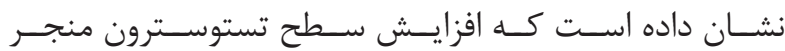

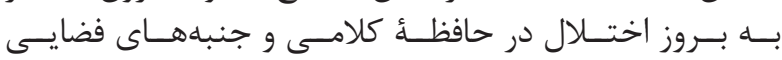

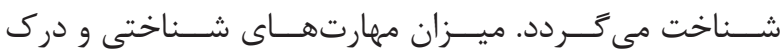

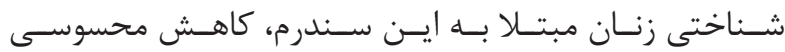

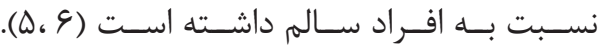

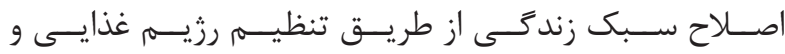

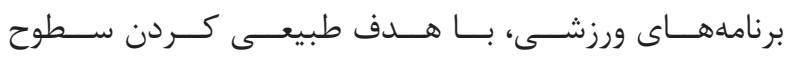

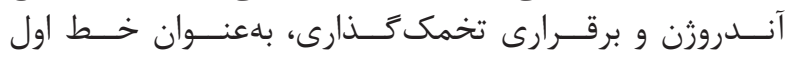

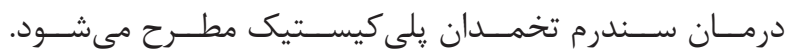

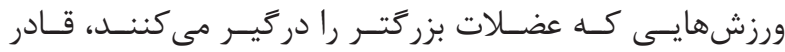

\footnotetext{
${ }^{7}$ Luteinizing hormone

${ }^{8}$ Hyperandrogenism

${ }^{9}$ Letrozole

${ }^{10}$ Rat

${ }^{11}$ Vaginal smear
} 
مــدت زمــان تأخيـر در رسـيدن بـــهـ سـكوى مخفــى و نيــز

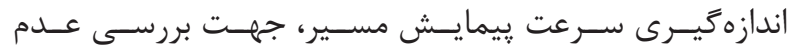

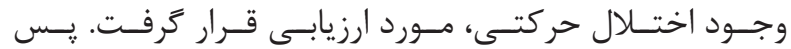

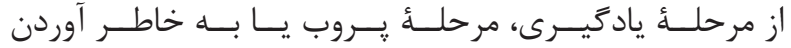

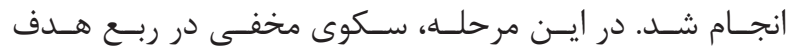

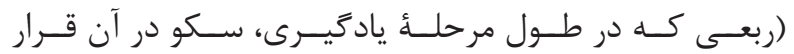

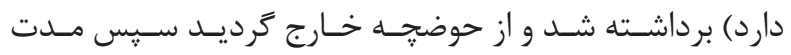

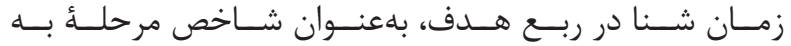

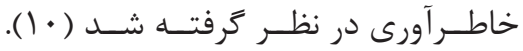

$$
\text { خونكَيرى و تهيئة سرم }
$$

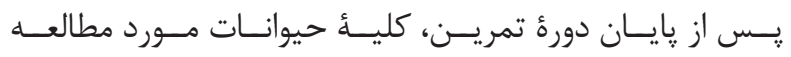

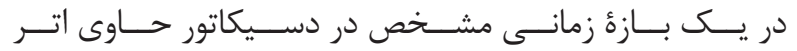

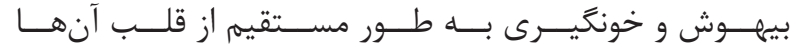

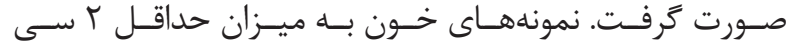

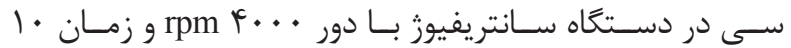

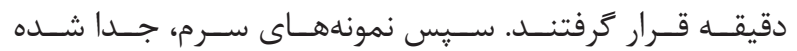

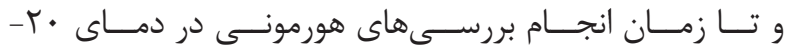

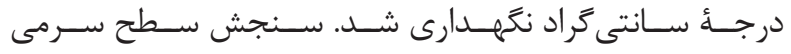

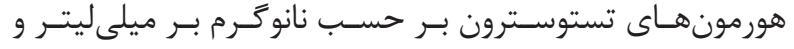

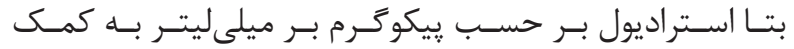
كيتهـاى الايـزا شـركت abcam (ab) A

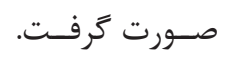

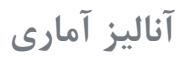

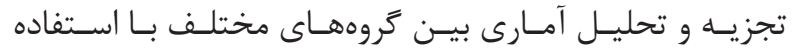

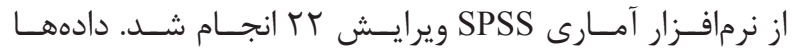

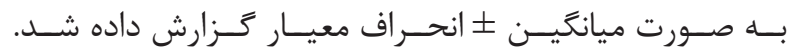

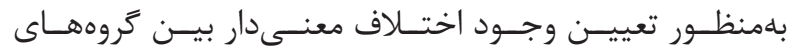

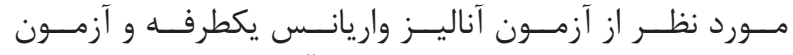

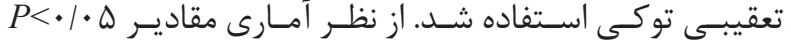

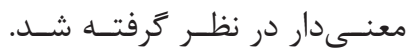

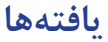

مشاهدة كيست هاى تخمدانى

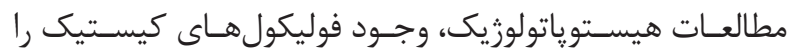

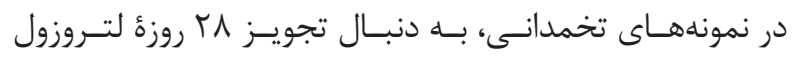

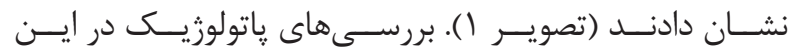

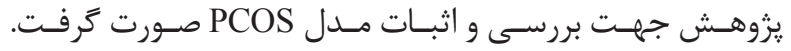

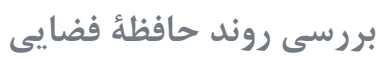

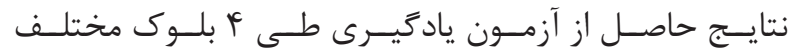

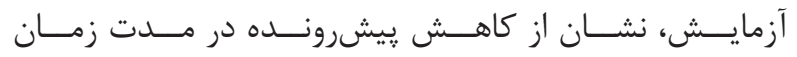

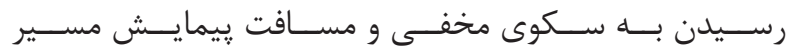

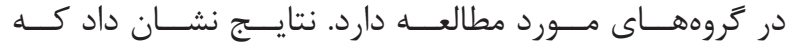

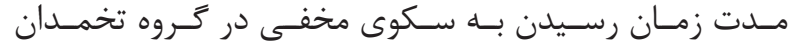

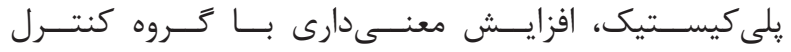

${ }^{12}$ Persistent vaginal cornification

${ }^{13}$ Haematoxylin

${ }^{14}$ Eosin

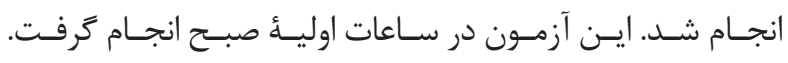

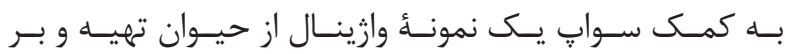

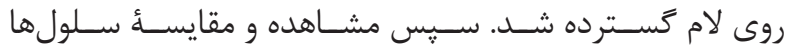

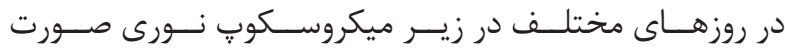

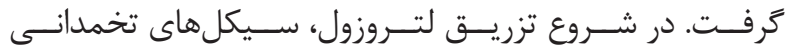

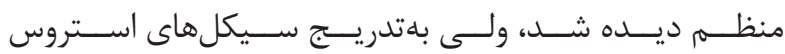

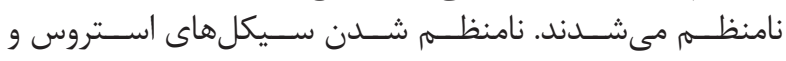

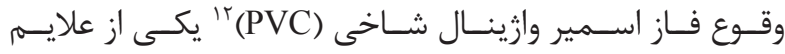

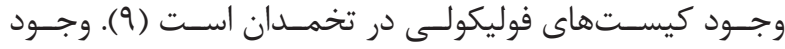

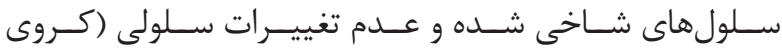

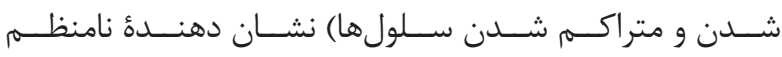

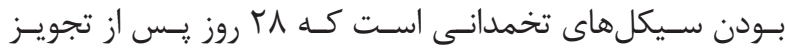

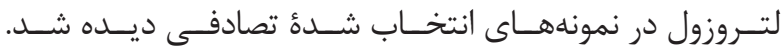

$$
\text { اثبات مدل با روش هيستو ياتولوزى }
$$

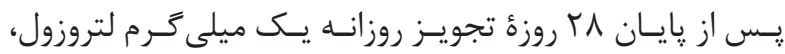

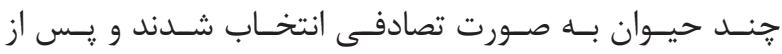

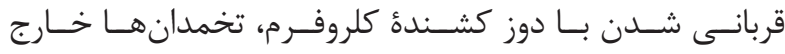

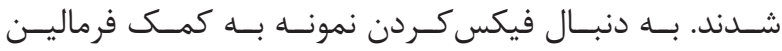

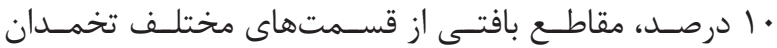

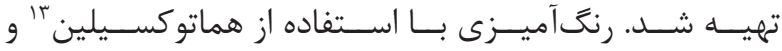

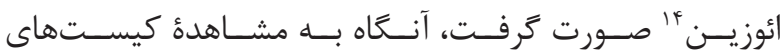

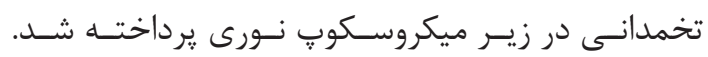

يروتكل تمرين شنا تصدان

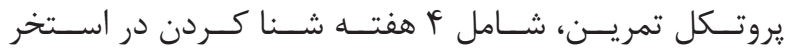

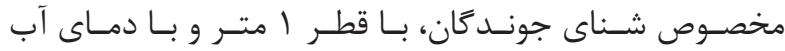
جـ

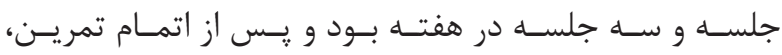

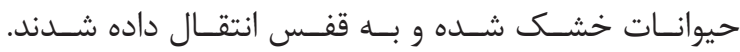
آزمون ماز آبى موريس حئس

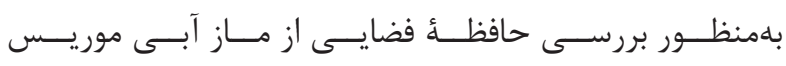

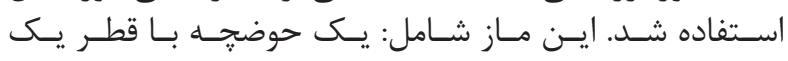

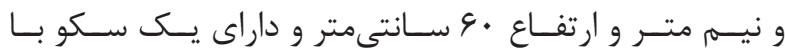

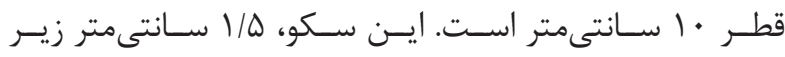

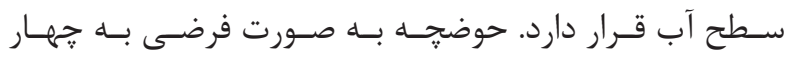

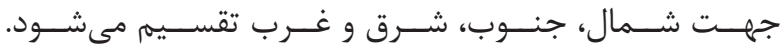

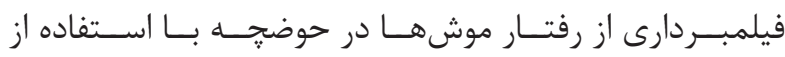

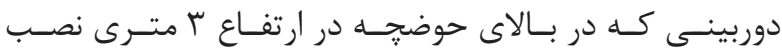

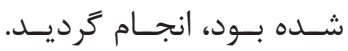

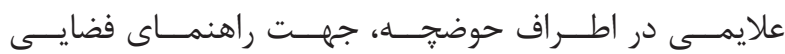

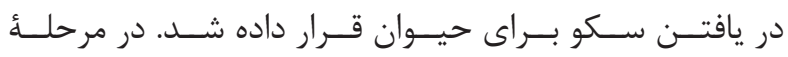

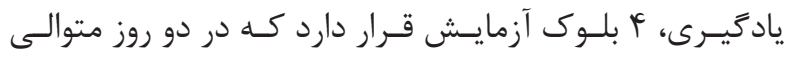

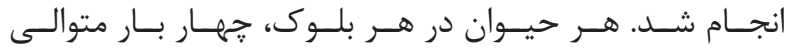

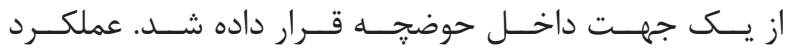

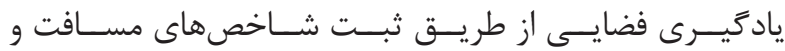




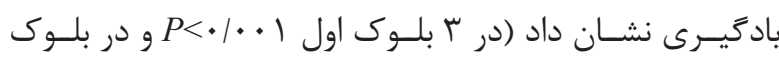

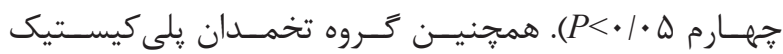

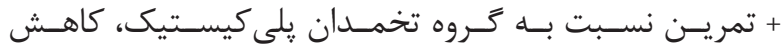

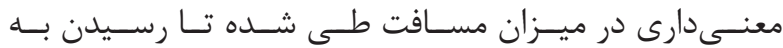

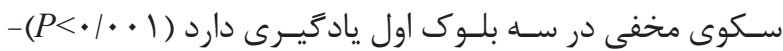

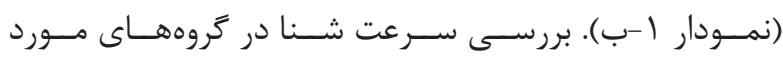

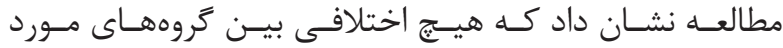

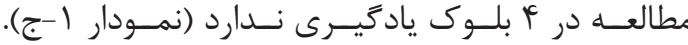

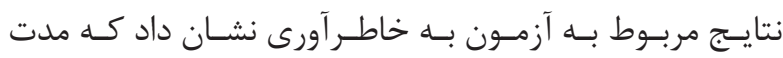

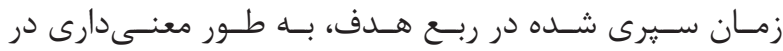

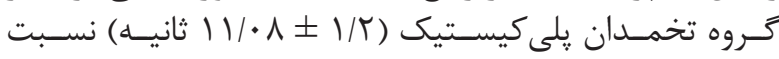

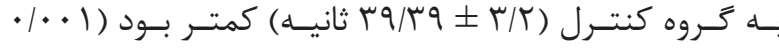

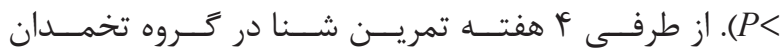

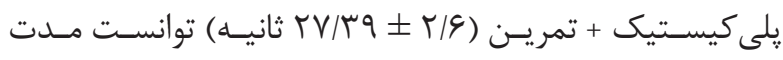

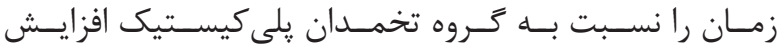

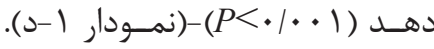

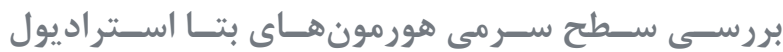

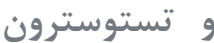

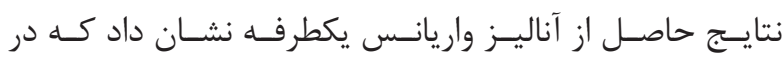

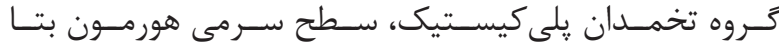
اسـتراديول (
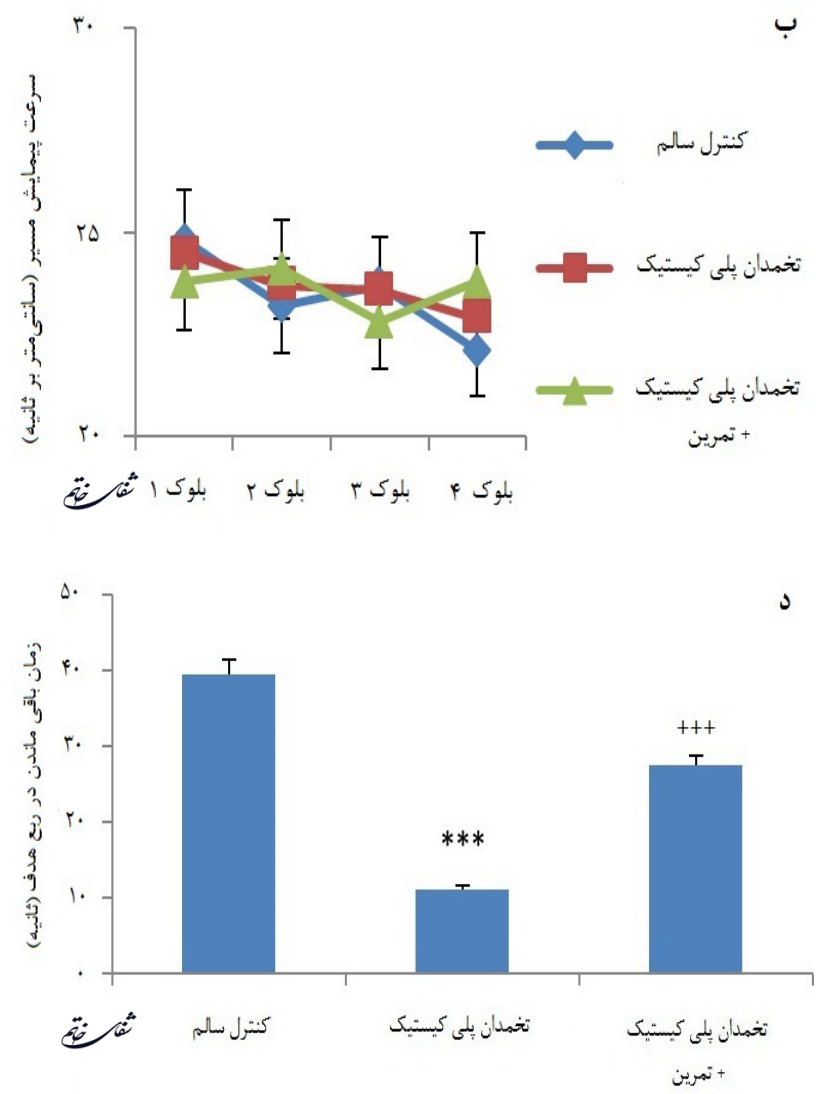

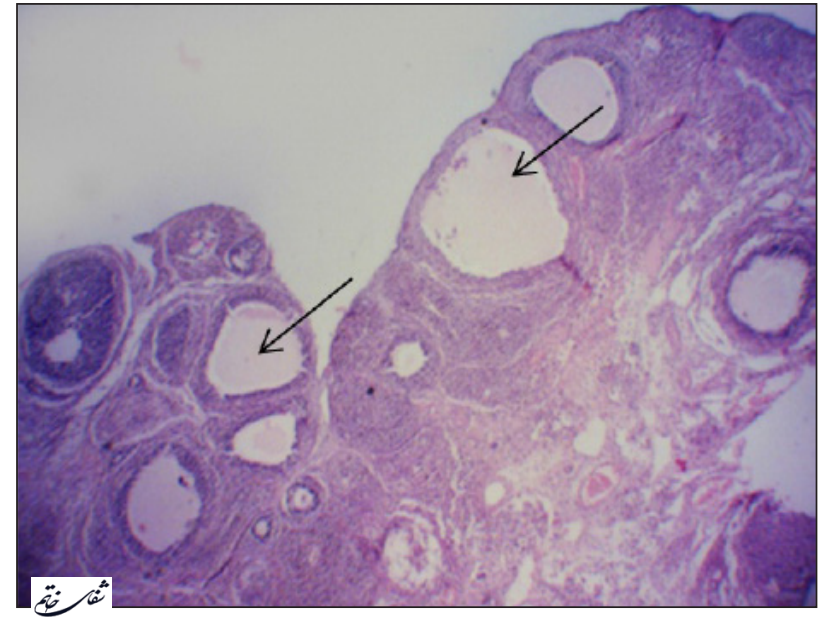

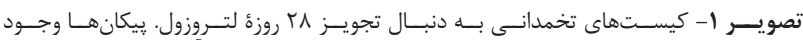

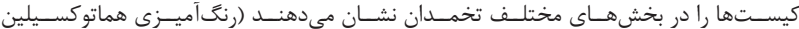

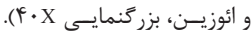

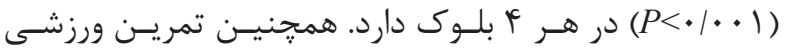

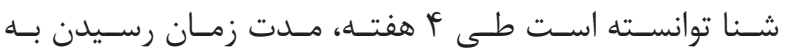

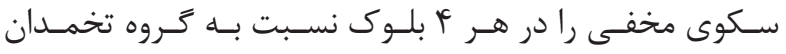

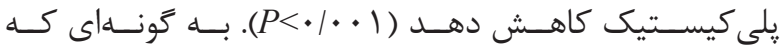

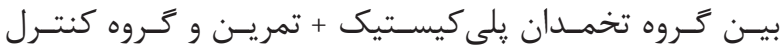

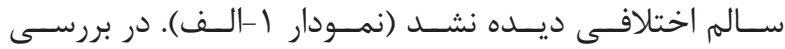

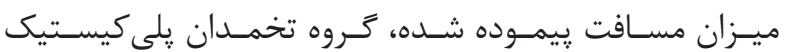

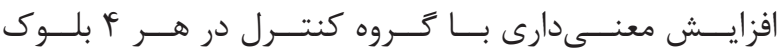
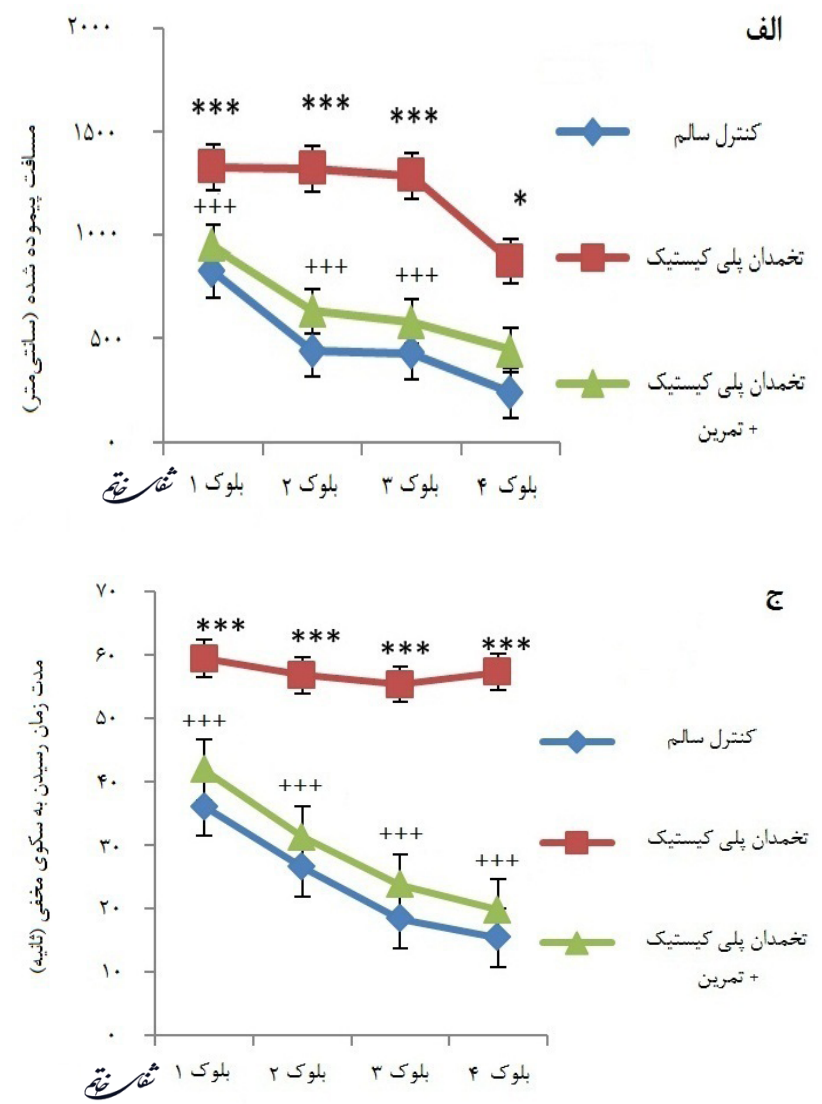

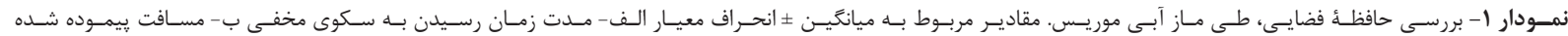

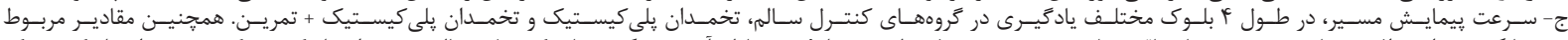

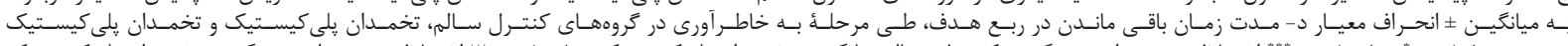

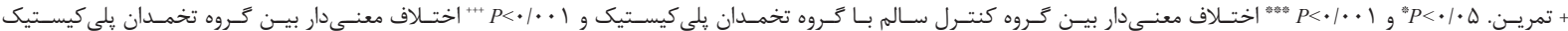



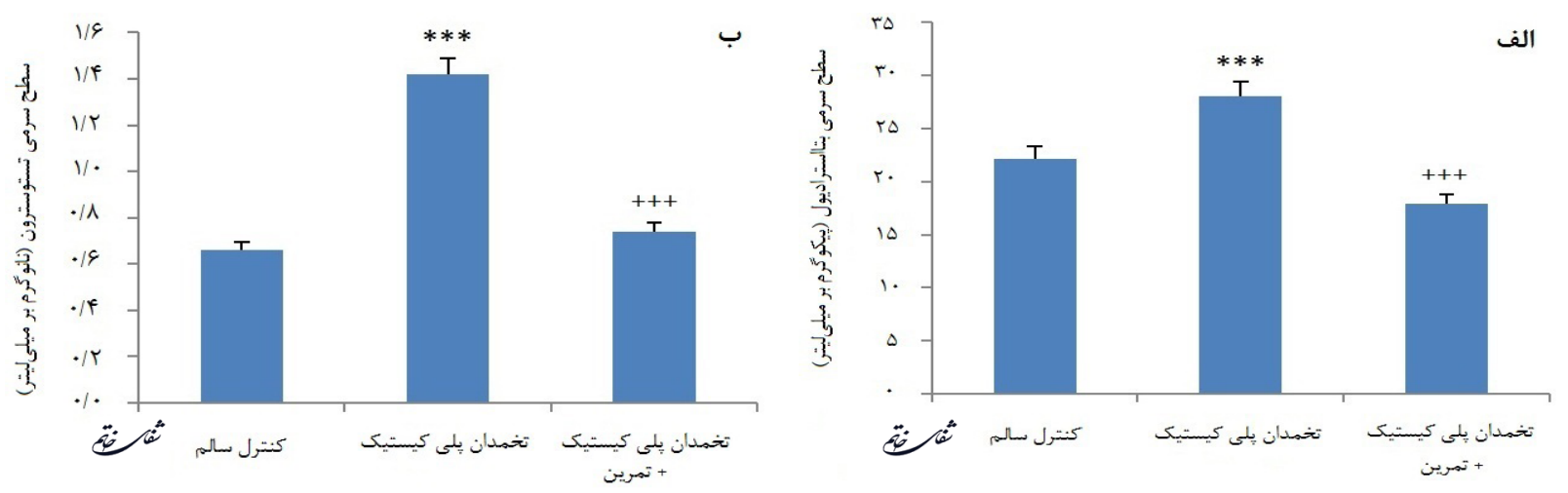

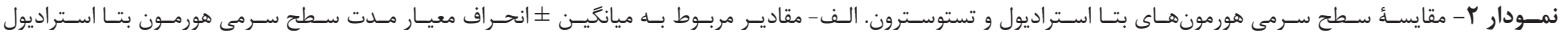

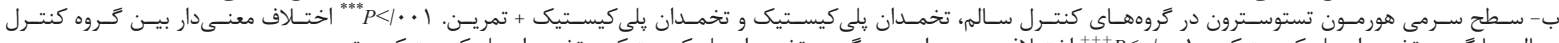

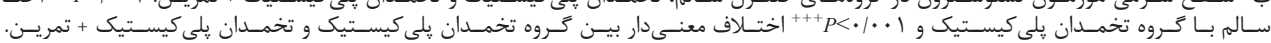

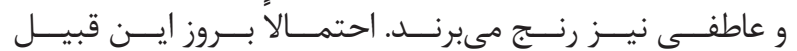

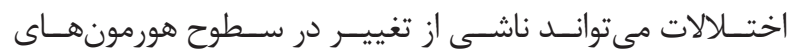

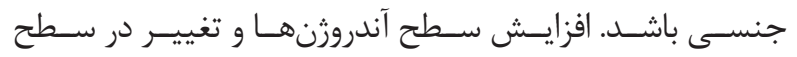

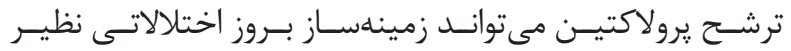

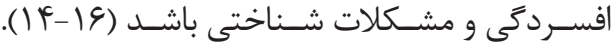

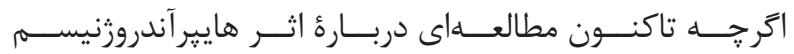

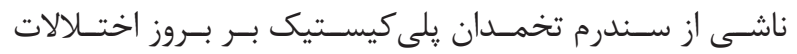

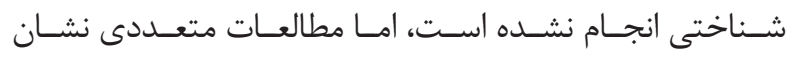

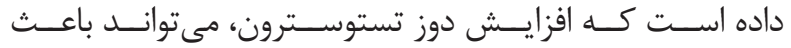

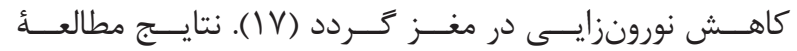

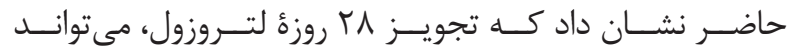

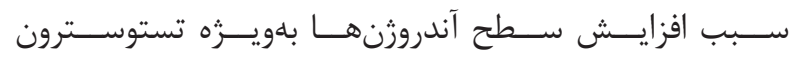

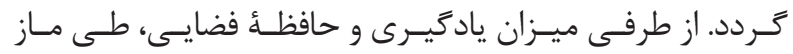

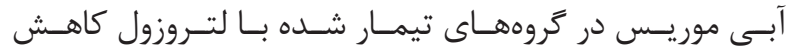

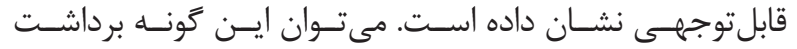

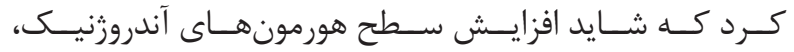

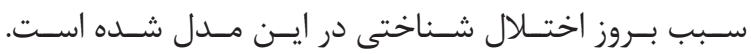

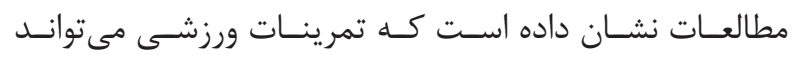

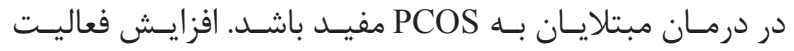

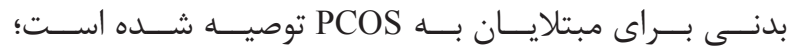

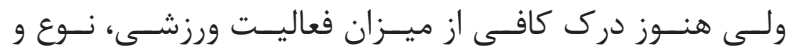

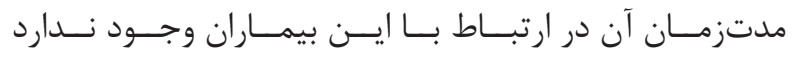

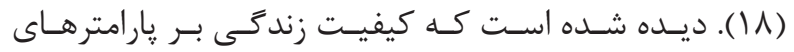

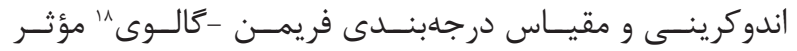

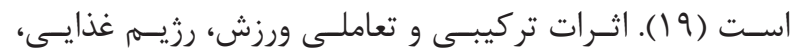

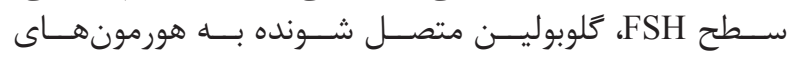

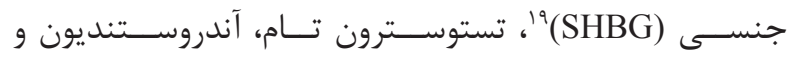

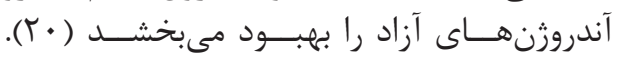

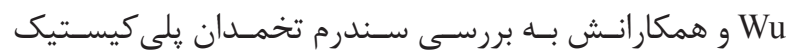

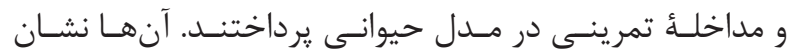

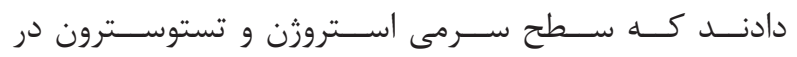

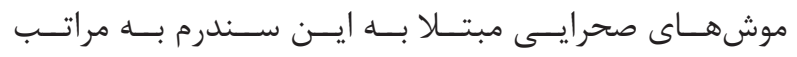

${ }^{15}$ Etiology

${ }^{16}$ Androstenedione

${ }^{17}$ Dehydroepiandrosterone sulfate

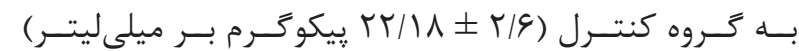

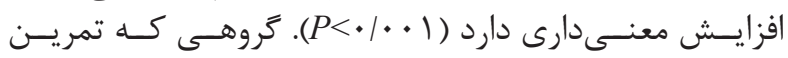

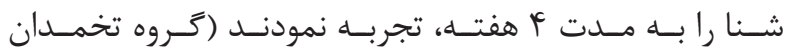

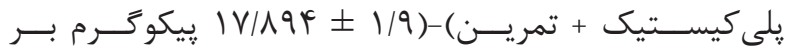

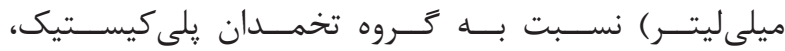

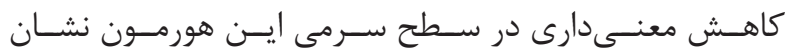

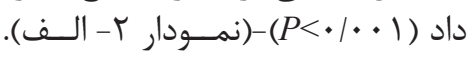

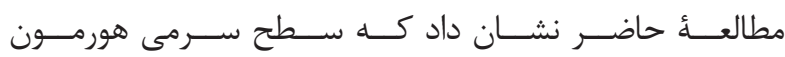

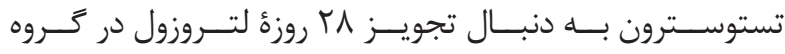

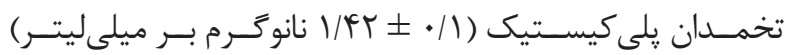

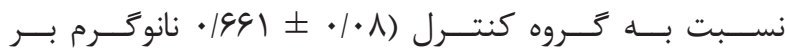

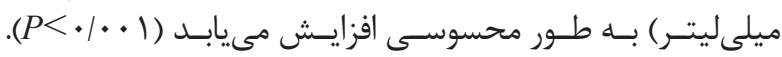

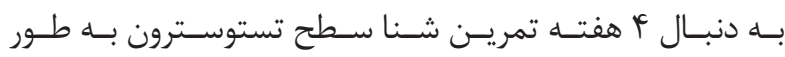

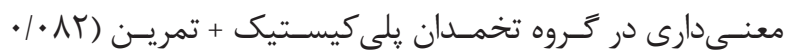

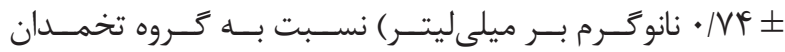

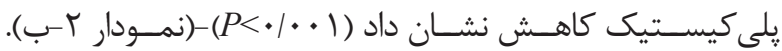

\section{بحث و نتيجه}

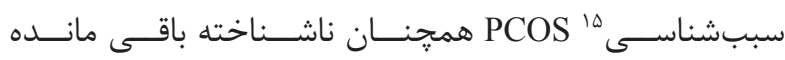

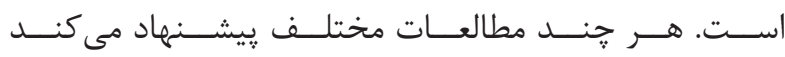

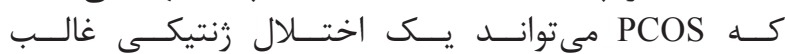

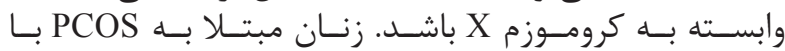

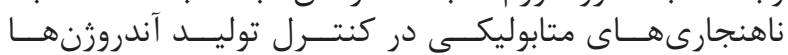

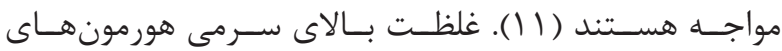

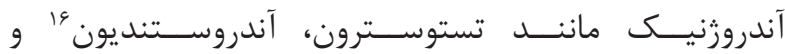

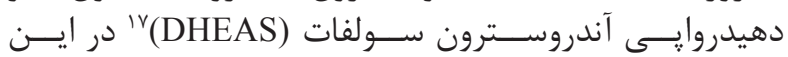

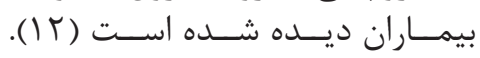

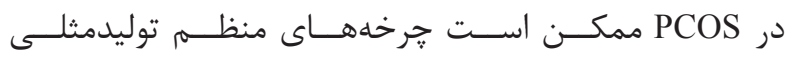

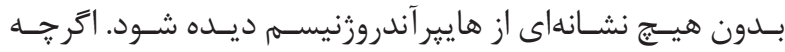

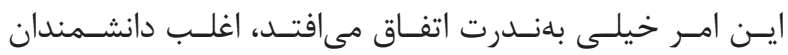

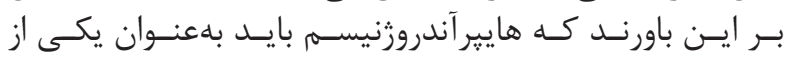

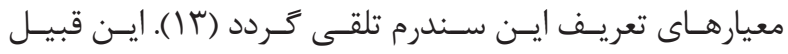

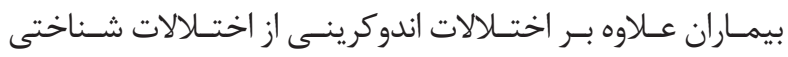

${ }^{18}$ Ferriman-Gallwey

${ }^{19}$ Sex hormone binding globulin 


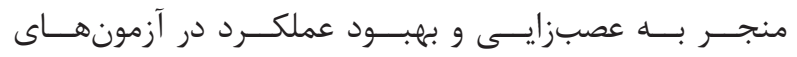

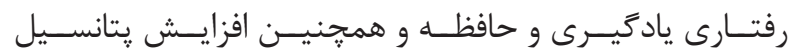

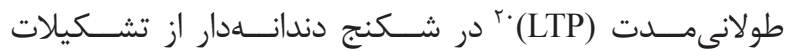

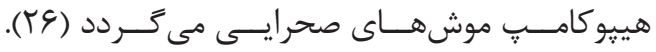

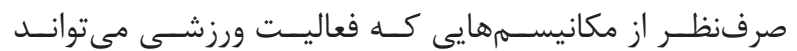

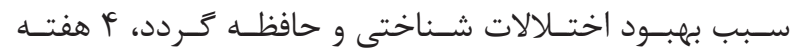

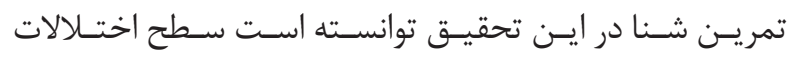

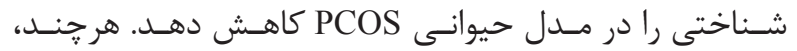

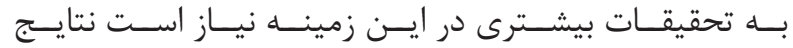

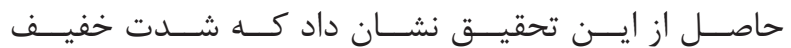

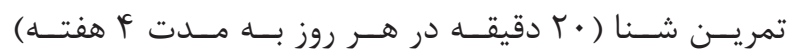

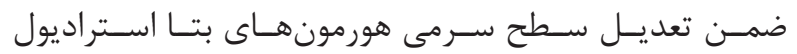

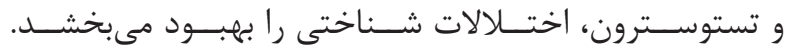

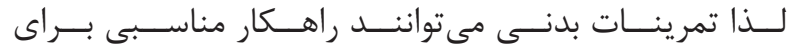

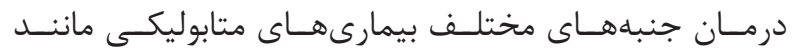

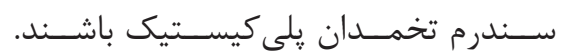
تشكر و قدردانى

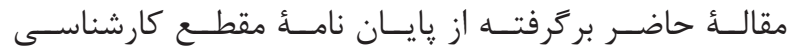

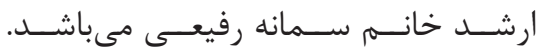

1. Caminos JE, Nogueiras R, Gaytán F, Pineda R, González CR, Barreiro ML. Novel expression and direct effects of adiponectin in the rat testis. Endocrinology. 2008; 149(7): 3390-402.

2. Moran LJ, Pasquali R, Teede HJ, Hoeger KM, Norman RJ. Treatment of obesity in polycystic ovary syndrome: a position statement of the androgen excess and polycystic ovary syndrome society. Fertil Steril. 2009; 92(6): 1966-82.

3. Thomson RL, Buckley JD, Noakes M, Clifton PM, Norman RJ, Brinkworth GD. The effect of a hypocaloric diet with and without exercise training on body composition, cardiometabolic risk profile, and reproductive function in overweight and obese women with polycystic ovary syndrome. J Clin Endocrinol Metab. 2008; 93(9): 3373-80.

4. Ehrmann DA. Polycystic ovary syndrome. N Engl J Med. 2005; 352(12): 1223-36.

5. Schattmann L, Sherwin BB. Testosterone levels and cognitive functioning in women with polycystic ovary syndrome and in healthy young women. Horm Behav. 2007; 51(5): 587-96.

6. Wolf OT, Kirschbaum C. Endogenous estradiol
بيشــتر از موش هــاى صحرايسـى ســالم مىباشــد ( (T). همانـــد مطالعــُ حاضـر، در مطالعـــ Al-Nozha و همكارانش نيز

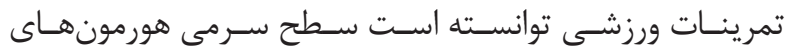

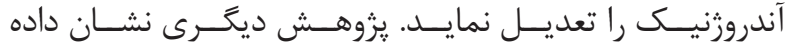

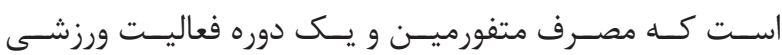

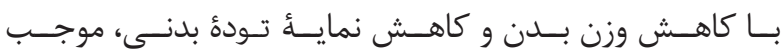

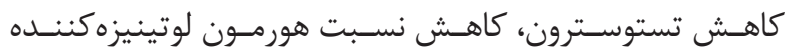

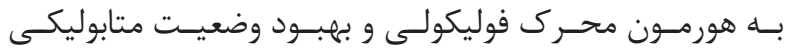

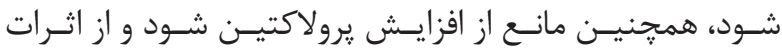

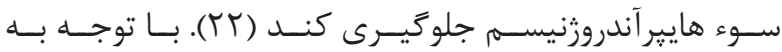

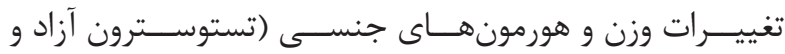

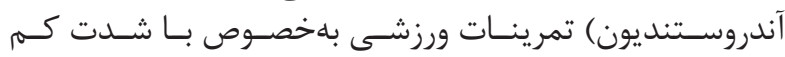

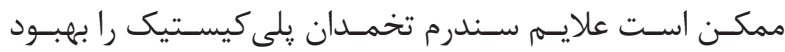

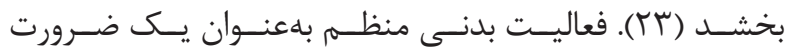

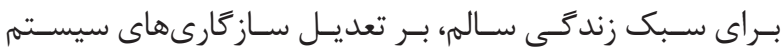

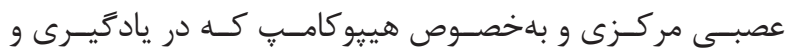

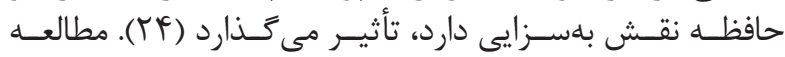

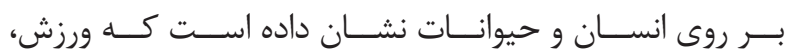

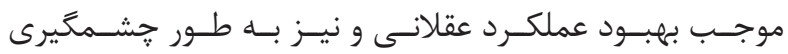

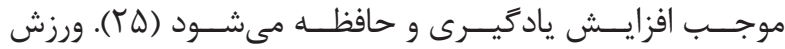

\section{منابع}

and testosterone levels are associated with cognitive performance in older women and men. Horm Behav. 2002; 41(3): 259-66.

7. Mannerås L, Cajander S, Lönn M, Stener-Victorin E. Acupuncture and exercise restore adipose tissue expression of sympathetic markers and improve ovarian morphology in rats with dihydrotestosterone-induced PCOS. Am J Physiol Regul Integr Comp Physiol. 2009; 296(4): R1124-31.

8. Mannerås L, Cajander S, Holmäng A, Seleskovic Z, Lystig T, Lönn M, et al. A new rat model exhibiting both ovarian and metabolic characteristics of polycystic ovary syndrome. Endocrinology. 2007; 148(8): 3781-91.

9. Schulster A, Farookhi R, Brawer JR. Polycystic ovarian condition in estradiol valerate-treated rats: spontaneous changes in characteristic endocrine features. Biol Reprod. 1984; 31(3): 587-93.

10. Fujimoto ST, Longhi L, Saatman KE, McIntosh TK. Motor and cognitive function evaluation following experimental traumatic brain injury. Neurosci Biobehav Rev. 2004; 28: 365-78.

11. Maharaj A. Polycystic ovary syndrome. JEMDSA.

${ }^{20}$ Long-term potentiation 
2009; 14(2): 86-95.

12. Lakhani K, Prelevic GM, Seifalian AM, Atiomo WU, Hardiman P. Polycystic ovary syndrome, diabetes and cardiovascular disease: risks and risk factors. J Obstet Gynaecol. 2004; 24(6): 613-21.

13. Azziz R, Carmina E, Dewailly D, DiamantiKandarakis E, Escobar-Morreale HF, Futterweit W, et al. Position statement: criteria for defining polycystic ovary syndrome as a predominantly hyperandrogenic syndrome: an Androgen Excess Society guideline. J Clin Endocrinol Metab. 2006; 91(11): 4237-45.

14. Barry JA, Parekh HS, Hardiman PJ. Visual-spatial cognition in women with polycystic ovarian syndrome: the role of androgens. Hum Reprod. 2013; 28(10): 2832-7.

15. Schattmann L, Sherwin BB. Effects of the pharmacologic manipulation of testosterone on cognitive functioning in women with polycystic ovary syndrome: a randomized, placebo-controlled treatment study. Horm Behav. 2007; 51(5): 579-86.

16. Kolahi L, Asemi N, Mirzaei M, Adibi N, Beiraghdar M, Maghami Mehr A. The relationship between quality of life and coping strategies in polycystic ovary syndrome patients. Adv Biomed Res. 2015; 4: 168. doi: 10.4103/2277-9175.162545.

17. SpritzerMD, Ibler E, Inglis W, Curtis MG. Testosterone and social isolation influence adult neurogenesis in the dentate gyrus of male rats. Neuroscience. 2011; 195: 180-90.

18. Moran LJ, Ranasinha S, Zoungas S, McNaughton SA, Brown WJ, Teede HJ. The contribution of diet, physical activity and sedentary behaviour to body mass index in women with and without polycystic ovary syndrome. Hum Reprod. 2013; 28(8): 2276-83.
19. Murri M, Luque-Ramırez M, Insenser M, OjedaOjeda M, Escobar-Morreale HF. Circulating markers of oxidative stress and polycystic ovary syndrome (PCOS): a systematic review and meta-analysis. Hum Reprod Update. 2013; 19(3): 268-88.

20. Harrison CL, Lombard CB, Moran LJ, Teede HJ. Exercise therapy in polycystic ovary syndrome: a systematic review. Hum Reprod Update. 2011; 17(2): 171-83.

21.Wu C, Lin F, Qiu S, Jiang Z. The characterization of obese polycystic ovary syndrome rat model suitable for exercise intervention. PLoS One. 2014; 9(6): e99155. doi: 10.1371/journal.pone.0099155.

22. Al-Nozha O, Habib F, Mojaddidi M, El-Bab MF. Body weight reduction and metformin: roles in polycystic ovary syndrome. Pathophysiology. 2013; 2(20):131-7 .

23. Miri M, Karimi Jashni H, Alipour F. Effect of exercise intensity on weight changes and sexual hormones (androstenedione and free testosterone) in female rats with estradiol valerate-induced PCOS. J Ovarian Res. 2014; 7: doi: 10.1186/17572215-7-37.

24. Cotman CW, Berchtold NC, Christie LA. Exercise builds brain health: key roles of growth factor cascades and inflammation. Trends Neurosci. 2007; 30 (9): 464-72.

25. Suto D, Akiyama K. Regulation of brain function by exercise. Neurobiol Dis. 2003; 13(1): 1-14.

26. Farmer J, Zhao X, van Praag H, Wodtke K, Gage FH, Christie BR. Effects of voluntary exercise on synaptic plasticity and gene expression in the dentate gyrus of adult male Sprague-Dawley rats in vivo. Neuroscience. 2004; 124(1): 71-9. 